\title{
Role of Elm1 in cell wall integrity and virulence of Candida glabrata
}

Ito $\mathrm{Y}^{1,2)}$, Miyazaki $\mathrm{T}^{1,3)}$, Nakayama $\mathrm{H}^{4)}$, Morita $\mathrm{A}^{4)}$, Tanaka $\mathrm{Y}^{5)}$, Takazono $\mathrm{T}^{1)}$, Saijo $\mathrm{T}^{1)}$, Shimamura $\mathrm{S}^{1)}$, Yamamoto $\mathrm{K}^{1}$, Imamura $\mathrm{Y}^{1}$ ), Izumikawa $\mathrm{K}^{3}$ ), Yanagihara $\mathrm{K}^{6}$ ), Kohno $\mathrm{S}^{1}$ ), Mukae $\mathrm{H}^{1,2)}$

1) Second Department of Internal Medicine, Nagasaki University Hospital

2) Department of Respiratory Medicine, Nagasaki University Graduate School of Biomedical Sciences

3) Department of Infectious Diseases, Nagasaki University Graduate School of Biomedical Sciences

4) Faculty of Pharmaceutical Sciences, Suzuka University of Medical Science

5) Department of Infection and Host Defense, Tohoku Medical and Pharmaceutical University

6) Department of Laboratory Medicine, Nagasaki University Hospital

Correspondence

Yuya Ito, MD

E-mail : y-ito@nagasaki-u.ac.jp

\section{Introduction}

The calcineurin signaling pathway plays critical roles in antifungal resistance and virulence in pathogenic fungi and has attracted attention as a novel target for antifungal therapy (1).

The serine/threonine protein kinase Elm1 is regulated by calcineurin and involved in multiple cellular functions, including mitosis, cytokinesis, and morphogenesis in Saccharomyces cerevisiae $(2,3)$. In this study, we performed functional analysis of Elm1 in the clinically important fungal pathogen Candida glabrata.

\section{Methods}

The deletion and reintroduction of ELM1 was performed in C. glabrata as described previously (1). The major cell wall components were measured using alkali-insoluble cell wall fraction (4). Antifungal susceptibility was examined using a commercially prepared colorimetric panel, Sensititre YeastOne microtiter panel. Virulence of the $\Delta e l m 1$ strain was examined using Galleria mellonella infection model (5). In this model, C. glabrata cells $\left(2.0 \times 10^{6}\right.$ cells / larva) were injected into the hemocoel through the last left pro-leg of larvae and survival was monitored daily for 7 days.

\section{Results}

WT

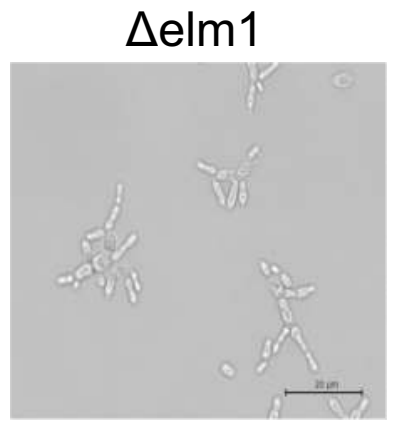

$\Delta$ elm1+ELM1

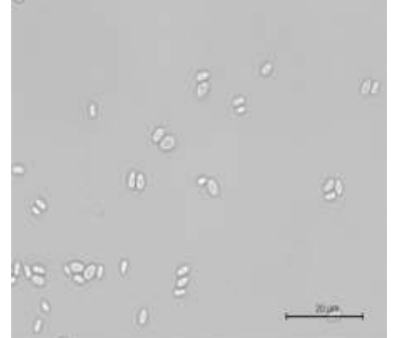

Fig 1. Cell morphology The $\Delta e / m 1$ strain displayed elongated morphology.
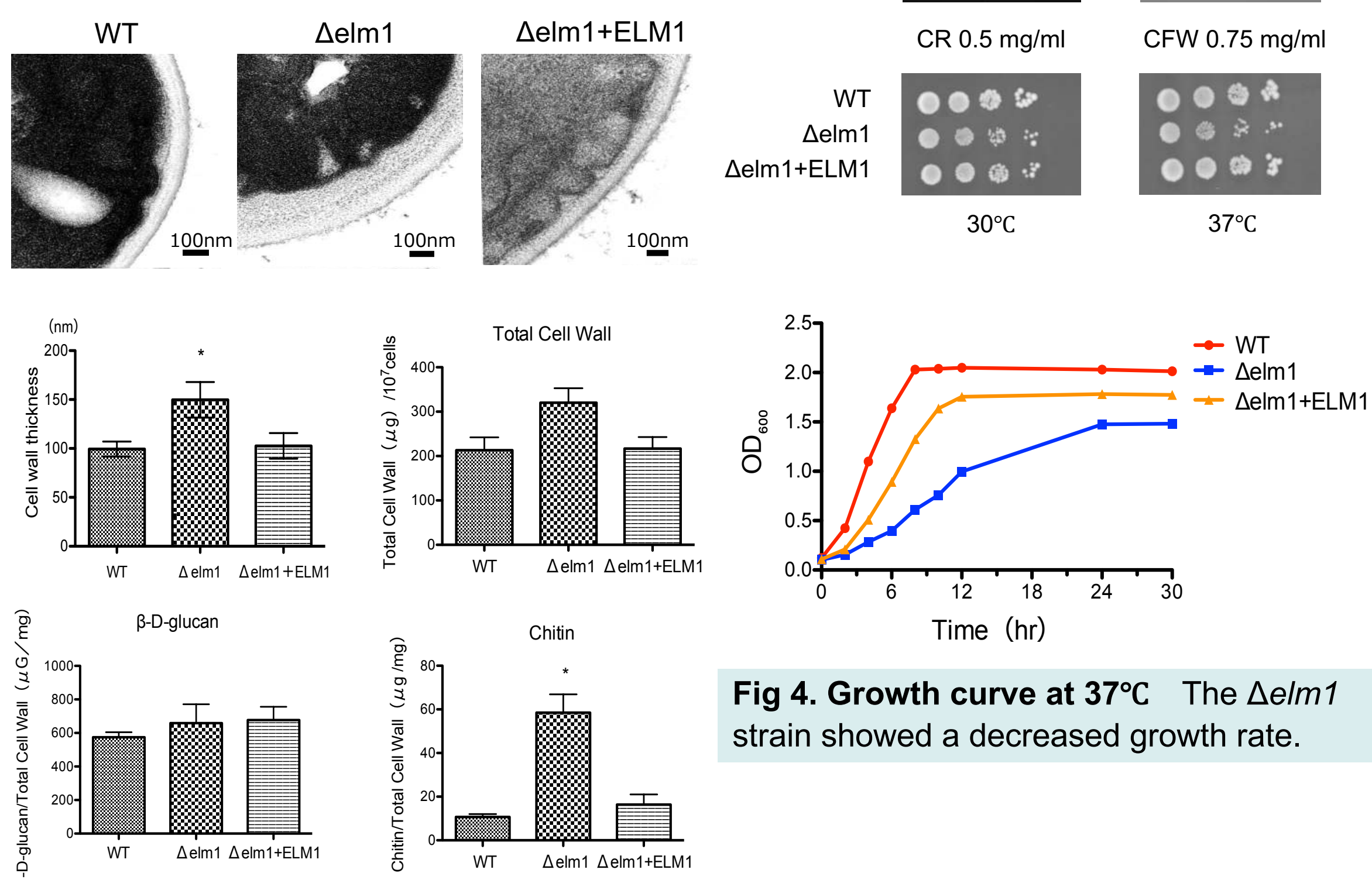

Fig 4. Growth curve at $37^{\circ} \mathrm{C}$ The $\Delta e / m 1$ strain showed a decreased growth rate.

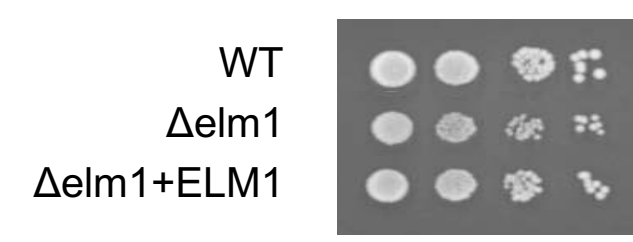

$10^{4} 10^{3} 10^{2} 10 \quad 10^{4} 10^{3} 10^{2} 10$
Control

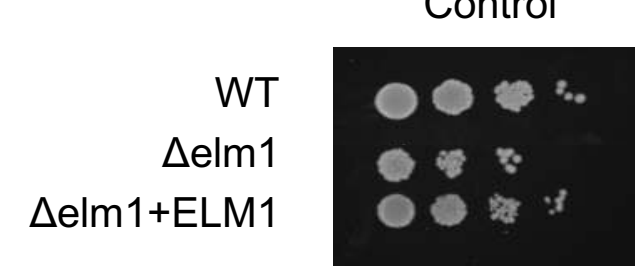

$\mathrm{CR} 0.5 \mathrm{mg} / \mathrm{ml}$

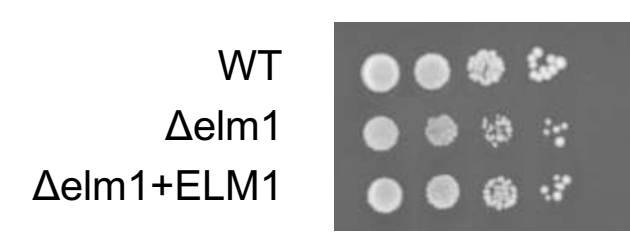

$30^{\circ} \mathrm{C}$

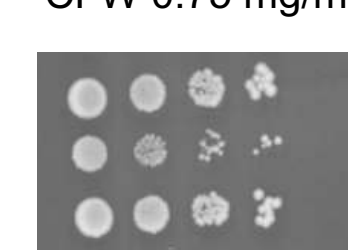

$37^{\circ} \mathrm{C}$

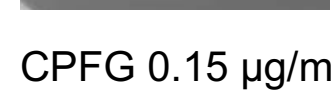

Fig.3 Spot dilution assay The $\Delta e l m 1$ strain increased sensitivity to high temperature and cell wall damaging agents including echinocandins, Congo red (CR), and calcofluor white (CFW).

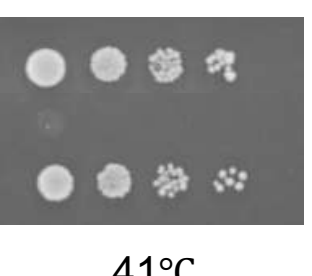

$41^{\circ} \mathrm{C}$
Fig 2. Cell wall thickness and component content The $\Delta e l m 1$ strain had significantly thicker cell wall and increased chitin content compared with the wild-type and ELM1-reconstituted strains.

$\left({ }^{*} p<0.01\right.$ one-way ANOVA)

Table 1. Antifungal susceptibility

\begin{tabular}{lccccc}
\hline \multirow{2}{*}{ Strain } & \multicolumn{5}{c}{ MIC $(\mu \mathrm{g} / \mathrm{ml})$} \\
\cline { 2 - 6 } & AND & MCF & CPF & VRC & AMB \\
\hline WT & 0.12 & 0.03 & 0.12 & 0.25 & 0.5 \\
$\Delta$ elm1 & 0.12 & 0.015 & 0.06 & 0.25 & 0.25 \\
$\Delta$ elm1 + ELM1 & 0.12 & 0.03 & 0.12 & 0.25 & 0.5 \\
\hline
\end{tabular}

AND, anidulafungin; $M C F$, micafungin; $C P F$, caspofungin

VRC, voriconazole; $\mathrm{AMB}$, amphotericin $\mathrm{B}$

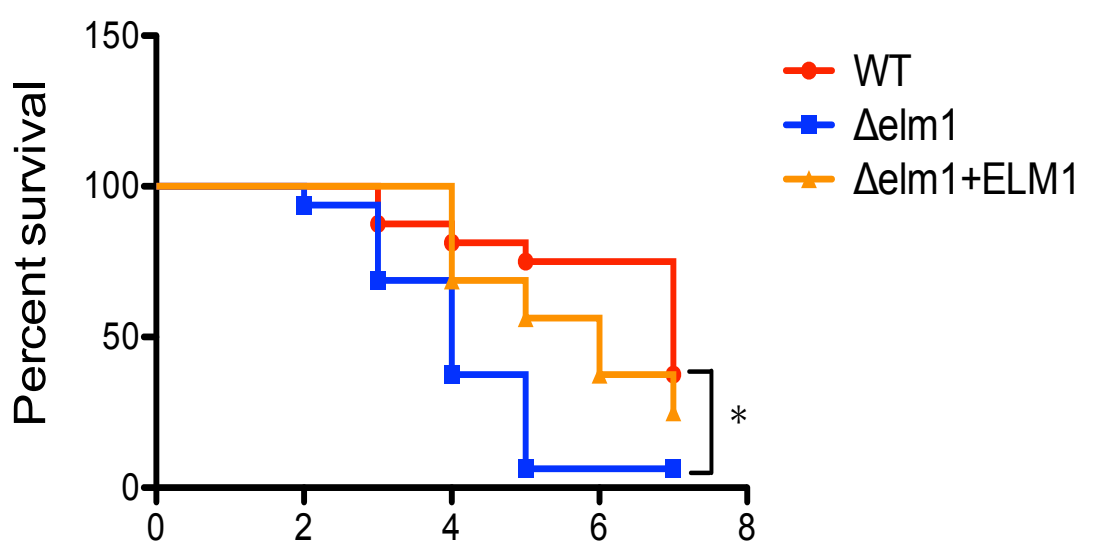

Days post-infection

Fig 5. Survival curve of $\mathbf{G}$ mellonella infected with C. glabrata.

The $\Delta e l m 1$ strain was more virulent than the wild-type strain

$\left({ }^{*} \mathrm{p}<0.01\right.$ Log-rank（Mantel-Cox) Test)

\section{Conclusions}

D The loss of Elm1 in C. glabrata resulted in elongated cell morphology, thicker cell wall with increased chitin content, decreased growth capacity, and increased sensitivity to cell wall stresses in vitro, however, the $\Delta e / m 1$ strain was more virulent than wild-type in vivo.

To understand molecular basis of these phenomena, further analyses using an elm1 strain controlled by the tetracyclineregulatable promoter and an elm1 kinase-dead mutant are now in progress.

\section{References}

1) Miyazaki T, et al. Antimicrob Agents Chemotherapy 54: 1639-43, 2010.

2) Goldman A, et al. Mol Cell 55: 422-35, 2014.

3) Sreenivasan A, et al. Mol Cell Biol 19: 7983-94, 1999.

4) Tanaka $Y$, et al. PLoS One 11: e0161371, 2016.

5) Ames L, et al. Virulence 8: 1909-17, 2017. 